structions as "it can be seen that at the present time it is not known with any certainty . . ." where a simple "we don't know . . ." would do. I was also disappointed to find very little stress placed on the increasing evidence of links between QSOs and active galaxies.

Overall, the book would be of interest to its intended market, but as such it must face comparison with such accepted standard works as Shapley's Galaxies (revised by Paul Hodge only a few years ago), Baade's Evolution of

\section{Sense of time}

An Inventive Universe. By $\mathrm{K}$. G. Denbigh. Pp. 220. (Hutchinson: London, 1975.) £3.75.

DR Denbigh, Principal of Queen Elizabeth College, University of London, is a scientist who has managed to fulfil the duties of his post without abandoning his own academic activities. A distinguished thermodynamicist he has long had a profound interest in the subject of time, both in its philosophical and its scientific aspects. He has read widely and thought deeply about it and other fundamental concepts in science. Since he also has the gift of writing clearly and concisely, his latest book, which is addressed to a wider audience than most of his previous publications, deserves a warm welcome and it is to be hoped that many will read it.

Denbigh believes, in the widest sense of the term, in the evolutionary nature of the universe. Although he never refers to A. N. Whitehead specifically, he can be regarded in this respect, at least, as one of his followers. Whitehead maintained that our whole conception of knowledge has been vitiated by the assumption, going back to the Greeks, that transition and creation are 'inferior' to changelessness and invariance.

In Denbigh's short book there are five chapters of about 175 pages in all, supplemented by some 35 pages of notes and references. The first, and longest, chapter is on our construction of the concept of time, which he points out depends specifically on mental processes, despite its intersubjective character. Much of physics, however, is based on what he calls "a sort of pared down time concept", involving only the notion of a linear sequence and a quantitative scale. Consequently there is considerable divergence of opinion concerning the anthropomorphic nature, or otherwise, of time's arrow and time's transience. Although Denbigh recognises the inadequacy of the B-theory standpoint (to use McTaggart's wellknown terminology), which denies objectivity to the distinctions we make
Stars and Galaxies and The Milky Way. by Bok and Bok. As a basic guide to galaxies for someone with an interest in astronomy, Mitton's book has no major flaws; unfortunately, however, when looked at against the opposition it is top of the second division rather than a big league competitor.

John Gribbin

John Gribbin is a Visiting Fellow at the Science Policy Research Unit, University of Sussex, UK.

between past, present and future, he takes the A-theorist Hans Reichenbach to task for using Heisenberg's uncertainty principle as the basis of an objective 'now'. Denbigh's own leanings are towards the A-theorists and he repudiates the claim of those $B$ theorists who cite relativity in their support, since there is nothing in relativity that necessitates the $\mathrm{B}$-theory standpoint. He concludes that the sense of time which we construct out of our conscious awareness is not confined to ourselves but provides an important link between the mental and the physical.

Chapters 2 and 3 are devoted, respectively, to dissipative processes and 'formative' processes. The former are associated with the law of increasing entropy, and the latter with the evolution of organisms and tissues of increasing complexity. These two distinct trends in nature are not incompatible for, whereas entropy is concerned with orderliness (and its decay), biological evolution is concerned with organisation. To elucidate the difference between these ideas Denbigh introduces the concept of "integrability", which he suggests may be analysed mathematically with the aid of graph theory. It is to be hoped that he will eventually find time to develop this stimulating train of thought more fully.

In Chapters 4 and 5, Denbigh discusses determinism and whether genuinely new things can come into existence during the course of time. $\mathrm{He}$ is a firm believer in the continual and natural evolution of novelty. At the end of the book he returns to the concept of time, stressing both its relation to consciousness and its intersubjective character. $\mathrm{He}$ argues that the totality of consciousness in the universe tends to increase, and he believes that a kind of principle of 'complementarity' (although he does not call it that) between the concepts of physics and chemistry and those pertaining to ourselves as 'persons' (rather than 'things') must be invoked if we are to understand the role of man in the world-order.

G. J. Whitrow

G. J. Whitrow is Professor of the History and Applications of Mathematics at Imperial College, University of London, UK.

\section{Immunological cookery}

Practical Immunology. By L. Hudson and F. C. Hay. Pp. xvi+298. (Blackwell Scientific: Oxford, London, Edinburgh and Melbourne, 1976.) £6.50.

THis book is required in the laboratories of all those who practise the mysteries of contemporary experimental immunology. It is clearly written, adequately illustrated and very useful.

The authors are research workers who have, in addition, considerable experience of teaching both practical and theoretical immunology. It seems from their book that they enjoyed what they were doing, that they did it well and wished to communicate their enthusiasm and competence to others.

In the contents list to the book there are a series of rather arbitrary headings such as "Initial preparations", "Cellular dynamics in vivo", "Antibody interaction with antigen", and "Advanced techniques in cellular immunology". But in fact the authors have compiled a list of the main ingredients of immunological cookery and then gone on to provide a series of recipes for their use. The emphasis is avowedly on the cellular components of immune responses as the authors believe that this represents a good starting point from which consideration of the humoral aspects of immunity can develop.

The title of the book is misleading in that it seems to embrace the whole practice of immunology, whereas it is restricted almost entirely to description of the practicalities of avant-garde experimentation. Thus there is hardly any consideration given to parasite immunology except, surprisingly, the Wasserman test: nor to histopathological evaluation of results.

This book, held in conjunction with that on The Immune System: a Course on the Molecular and Cellular Basis of Immunity by Hobart and McConnell (Blackwell, 1976) and the older title Essential Immunology by Roitt (1974) should equip any budding immunologist with a knowledge of what has been done, how it was accomplished and what it all means. All that would be required to complete this paper armamentarium would be a text on "What to do next in Immunology". That would be useful.

A. J. S. Davies

A. J. S. Davies is Professor of Immuno biology in the University of London working at the Chester Beatty Research Institute, London, UK. 